\title{
Increasing Mobility via In-hospital Ambulation Protocol Delivered by Mobility Technicians: A Pilot Randomized Controlled Trial
}

\author{
Aaron C Hamilton, MD, MBA ${ }^{1}$; Natalie Lee, MD, MPH²; Mary Stilphen, PT, DPT ${ }^{3}$; Bo Hu, PhD; \\ Sarah Schramm, MA ${ }^{5}$; Frederick Frost, $\mathrm{MD}^{6}$; Jacqueline Fox, RN5; Michael B Rothberg, MD, MPH ${ }^{5 *}$
}

\begin{abstract}
${ }^{1}$ Department of Hospital Medicine, Cleveland Clinic, Cleveland, Ohio; ${ }^{2}$ Medicine Institute, Cleveland Clinic, Cleveland, Ohio; ${ }^{3}$ Rehabilitation and Sports Therapy, Cleveland Clinic, Cleveland, Ohio; ${ }^{4}$ Department of Quantitative Health Sciences, Cleveland Clinic, Cleveland, Ohio; ${ }^{5}$ Center for Value-Based Care Research, Medicine Institute, Cleveland Clinic, Cleveland, Ohio; ${ }^{6}$ Physical Medicine and Rehabilitation, Cleveland Clinic, Cleveland, Ohio.
\end{abstract}

BACKGROUND: Ambulating medical inpatients may improve outcomes, but this practice is often overlooked by nurses who have competing clinical duties.

OBJECTIVE: This study aimed to assess the feasibility and effectiveness of dedicated mobility technician-assisted ambulation in older inpatients.

DESIGN: This study was a single-blind randomized controlled trial.

SETTING: Patients aged $\geq 60$ years and admitted as medical inpatients to a tertiary care center were recruited.

INTERVENTION: Patients were randomized into two groups to participate in the ambulation protocol administered by a dedicated mobility technician. Usual care patients were not seen by the mobility technician but were not otherwise restricted in their opportunity to ambulate.

MEASUREMENTS: Primary outcomes were length of stay and discharge disposition. Secondary outcomes included change in mobility measured by six-clicks score, daily steps measured by Fitbit, and 30-day readmission.

RESULTS: Control $(n=52)$ and intervention $(n=50)$ groups were not significantly different at baseline. Of patients randomized to the intervention group, $74 \%$ participated at least once. Although the intervention did not affect the primary outcomes, the intervention group took nearly $50 \%$ more steps than the control group ( $P$ $=.04)$. In the per protocol analysis, the six-clicks score significantly increased $(P=.04)$. Patients achieving $\geq 400$ steps were more likely to go home $(71 \%$ vs $46 \%, P=.01)$.

CONCLUSIONS: Attempted ambulation three times daily overseen by a dedicated mobility technician was feasible and increased the number of steps taken. A threshold of 400 steps was predictive of home discharge. Further studies are needed to establish the appropriate step goal and the effect of assisted ambulation on hospital outcomes. Journal of Hospital Medicine 2019;14:272-277. Published online first February 20, 2019. ( 2019 Society of Hospital Medicine $\mathrm{n}$ dividuals aged 65 years and over represent $13 \%$ of the United States population and account for nearly $40 \%$ of hospital discharges. ${ }^{1}$ Bedrest hastens the functional decline of older patients ${ }^{2-5}$ and is associated with risk of serious complications, such as falls, delirium, venous thrombosis, and skin breakdown. ${ }^{6,7}$ Ambulation is widely recognized as important for improving hospital outcomes. ${ }^{8-10}$ Observational studies suggest that increases of 600 steps per day are associated with shortened length of hospital stay. ${ }^{9}$ However, randomized trials of assisted ambulation have not demonstrated consistent benefit.11-14 As a result, usual care at most hospitals in the United States does not include assisted ambulation. Even when ambulation is ordered, execution of the orders is inconsistent. ${ }^{15-17}$

Studies have demonstrated the benefits of various exercise *Corresponding Author: Michael B. Rothberg, MD, MPH; E-mail: Rothbem@
ccf.org; Telephone: 216-445-6600, Twitter: @MRothbergMD

Received: July 26, 2018; Revised: December 7, 2018;

Accepted: December 20, 2018

๑) 2019 Society of Hospital Medicine DOI 10.12788/jhm.3153 protocols for older patients in rehabilitation facilities, ${ }^{18,19}$ medical intensive care units, ${ }^{20}$ and medical and surgical wards. ${ }^{13,18,21}$ These interventions are usually nursing centered; however, assisting patients with ambulation multiple times per day may be a burdensome addition to the myriad responsibilities of nurses. ${ }^{19,22,23}$ In fact, ambulation orders are the most frequently overlooked nursing task. ${ }^{24}$

We designed a graded protocol of assisted ambulation implemented by a dedicated patient care nursing assistant (PCNA) multiple times daily to increase patient mobility. The objective of this study was to assess the feasibility and effectiveness of such an intervention for older inpatients. We hypothesized that the intervention would prove feasible and improve hospital outcomes, including less need for inpatient rehabilitation and shorter length of stay.

\section{METHODS}

We conducted a single-blind randomized controlled trial of patients aged $\geq 60$ years and admitted as medical inpatients to the Cleveland Clinic Main Campus, a tertiary care center with over 1,440 inpatient beds. The consent form and study 
protocol were approved by the Cleveland Clinic Institutional Review Board, and the study was registered with ClinicalTrials. gov (NCT02757131).

\section{Patients}

All patients who were admitted to study wards for a medical illness and evaluated by Physical Therapy (PT) were eligible for the study. PT evaluations were ordered by the medical team if deemed necessary on the basis of factors, such as age, estimated mobility, and concerns raised by the ancillary staff. All patients who were expected to be discharged to a skilled nursing facility placement or who required home PT received a PT evaluation. Assessment of mobility was documented via Activity Measure for Postacute Care Inpatient Basic Mobility "six-clicks" short form, hereafter abbreviated as "six-clicks." Based on past experience, patients with scores $<16$ rarely go home ( $<20 \%$ of the time), and those with scores $>20$ usually go home regardless of ambulation. Therefore, only patients with scores of 16-20 were invited to participate in the study. Although patients who were not evaluated by PT might also benefit from the intervention, we required a six-clicks score to assess eligibility. The exclusion criteria included anticipated remaining length of stay less than three days, admission under observation status, admission to the intensive care unit $\left(I C U_{1}\right)$ patients receiving comfort care measures, and patients with medical conditions precluding ambulation, such as decompensated heart failure or unstable angina.

\section{Randomization}

Patients were randomized to "usual care" or "mobility technician" after baseline evaluation using a computerized system. A block randomization scheme with a size of four was used to ensure an approximately equal number of patients per group.

\section{Intervention}

Patients randomized to the intervention group were asked to participate in the ambulation protocol outlined by the PT three times daily under the supervision of the mobility technician. The protocol involved four exercise levels (sitting, standing, walking, and stairs), which were implemented depending on the patient's physical capacity. The mobility technicians, who were PCNAs, were trained by the PT team. PCNAs have no specific degrees or certification. They are taught safe handling techniques during their job orientation, so they already had an understanding of how to transfer and assist a patient with ambulation. The mobility technician training consisted of one four-hour session run by the PT team in the physical therapy department and the nursing unit. The training included safe handling practices and basic mobility, such as transfers from bed to chair, bed to standing, walking with assistance, and walking independently with equipment such as cane, rolling walker, and walking belt. All instruction was demonstrated by the trainer, and the mobility technician was then able to practice. The mobility technician then shadowed the trainer and practiced the techniques under supervision. Competency was assessed by the trainer.

The cohort of patients randomized to "usual care" was not seen by the mobility technicians but was not otherwise restrict- ed in nursing's baseline ability to execute recommendations placed by the PT team. Compliance with the recommendations is highly variable and dependent on patient acuity during the shift, staffing issues, and competing duties. Cleveland Clinic promotes a "culture of mobility," and nurses are encouraged to get patients out of bed and assist with ambulation.

\section{Study Instruments-Measures of Mobility}

The six-clicks instrument is a tool for measuring basic mobility. It was adapted from the Activity Measures for Post-Acute Care (AM-PAC) instrument. ${ }^{25}$ Although initially created for self-report in the post-acute care setting, six-clicks has been validated for use by PTs in the acute care setting ${ }^{26}$ and is currently in use at more than 1,000 US hospitals. Cleveland Clinic PTs have used this measure for routine evaluation since 2011. The instrument has high interrater reliability and can predict discharge disposition. ${ }^{27-29}$

Each patient was provided with a tracking device (Fitbit) attached at the wrist to record daily steps for measuring mobility. The use of Fitbit has been validated in ambulatory and inpatient settings ${ }^{30}$ The device produces step counts within $3 \%$ of the observed step count for most patients but may undercount steps in patients with very slow gait. ${ }^{31}$ The device was provided to each enrollee and collected at discharge.

\section{Variables}

Demographic information, comorbid diagnoses, and discharge destination were extracted from the electronic medical record. Information on prehospitalization physical activity level was obtained from the initial PT assessment. Falls were tracked through the safety event reporting system.

\section{Outcomes}

The primary outcomes were discharge disposition and hospital length of stay. The secondary outcomes included average steps per day, change in six-clicks score from admission to discharge, inpatient mortality, admission to ICU, falls, deep vein thrombosis, pulmonary embolism, or pneumonia, and readmission within 30 days.

\section{Statistical Analysis}

Patient characteristics were summarized as means and standard deviations or medians and interquartile ranges for continuous variables and as frequencies and percentages for categorical variables. The t-test or Wilcoxon rank sum test was applied to compare continuous characteristics between the intervention and control groups. Chi-squared test or Fisher's exact test was applied to compare categorical characteristics. Given its skewed distribution, the length of stay was log-transformed and compared between the two groups using Student's t-test. Chi-squared test was used to compare categorical outcomes. The analysis of final six-clicks scores was adjusted for baseline scores, and the least-square estimates are provided. A linear mixed effects model was used to compare the number of daily steps taken because each participant had multiple steps measured. Results were adjusted for prehospital activity. In addi- 
TABLE 1. Comparison of Baseline Characteristics between the Two Groups

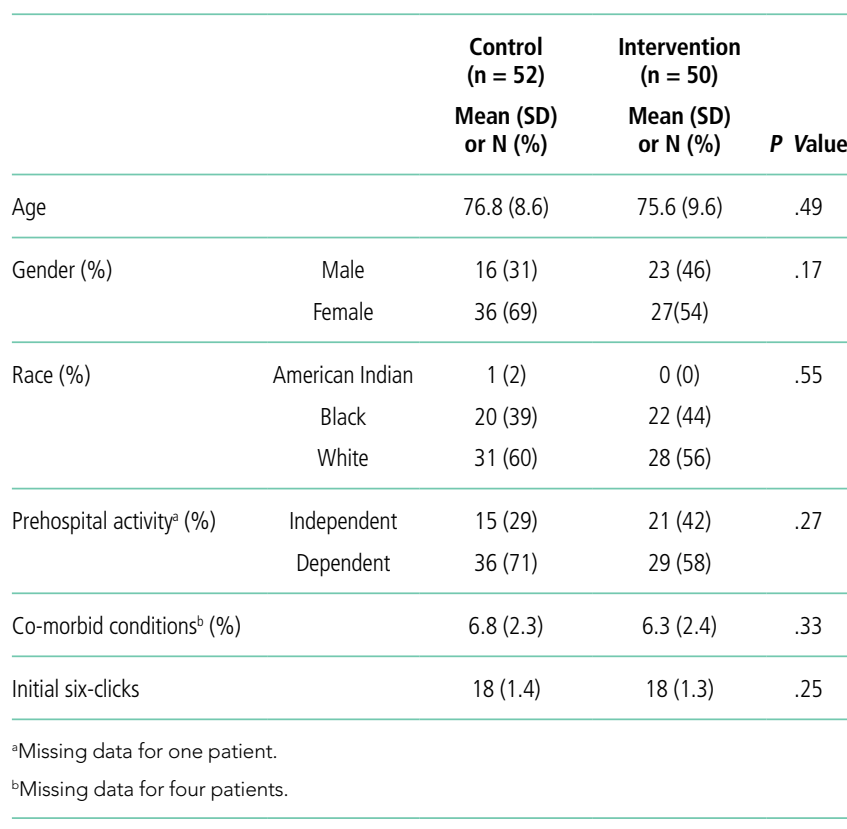

tion to comparing the total steps taken by each group, we determined the proportion of patients who exceeded a particular threshold by taking the average number of steps per day for all subjects and relating it to home discharge using the Receiver Operating Characteristics (ROC) curve. An optimal cut-off was determined to maximize the Youden index. We also compared the proportion of patients who exceeded 900 steps because this value was previously reported as an important threshold. ${ }^{32}$ All analyses were conducted using intention-to-treat principles. We also conducted a per-protocol analysis in which we limited the intervention group to those who received at least one assisted ambulation session. A dose-response analysis was also performed, in which patients were categorized as not receiving the therapy, receiving sessions on one or two days, or receiving them on more than two days.

All analyses were conducted using R-studio (Boston, MA). Statistical significance was defined as a $P$-value $<.05$. Given that this is a pilot study, the results were not adjusted for multiple comparisons.

\section{RESULTS}

Characteristics of patients in the intervention and control groups are shown in Table 1. The patients were mostly white and female, with an average age in the mid-70s (range 61-98). All measures evaluated were not significantly different between the intervention and control groups. However, more patients in the intervention group had a prehospital activity level classified as independent.

Table 2 demonstrates the feasibility of the intervention. Of patients randomized to the intervention group, 74\% were ambulated at least once. Once enrolled, the patients successfully participated in assisted ambulation for about two-thirds
TABLE 2. Implementation of the Intervention (restricted to 37 patients with any attempts) ${ }^{\text {a }}$

\begin{tabular}{|c|c|c|c|c|}
\hline Metric & Mean (SD) & Minimum & Maximum & Median [IQR] \\
\hline Days visited & $3.1(2.4)$ & 1 & 10 & $2[1,4]$ \\
\hline$\%$ days visited ${ }^{b}$ & $63(36)$ & 8 & 100 & $50[43,86]$ \\
\hline$\%$ days visited & $37(18)$ & 7 & 71 & $33[20,50]$ \\
\hline \# of attempts & $11(11)$ & 1 & 43 & $7[3,11]$ \\
\hline \# of successful attempts & $6(7.0)$ & 0 & 30 & $4[2,7]$ \\
\hline Total duration (in mins) & $66(106)$ & 0 & 591 & $31[17,66]$ \\
\hline \# of successful attempts ${ }^{c}$ & $6.5(7.0)$ & 1 & 30 & $4[2,7]$ \\
\hline Total duration (in mins) ${ }^{\mathrm{d}}$ & $72(109)$ & 9 & 591 & $32[19,68]$ \\
\hline
\end{tabular}

${ }^{a}$ One patient did not have discharge date due to death. ${ }^{b}$ Divided by the difference between discharge and Fitbit placement date; 11 subjects had a difference of one day, and one subject had a difference of 0 .

'Divided by length of stay

'Excludes three patients with no successful attempts

Abbreviation: IQR, interquartile range; $S D$, standard deviation.

of their hospital stay. However, the intervention was delivered for only one-third of the total length of stay because most patients were not enrolled on admission. On average, the mobility technicians made 11 attempts to ambulate each patient and $56 \%$ of these attempts were successful. The proportion of unsuccessful attempts did not change over the course of the study. The reasons for unsuccessful attempts included patient refusal $(n=102)$ or unavailability $(n=68)$, mobility technicians running out of time $(n=2)$, and other $(n=12)$.

Initially, the mobility technicians were not available on weekends. In addition, they were often reassigned to other duties by their nurse managers, who were dealing with staffing shortages. As the study progressed, we were able to secure the mobility technicians to work seven days per week and to convince the nurse managers that their role should be protected. Consequently, the median number [IOR] of successful attempts increased from $1.5[0,2]$ in the first two months to $3[0,5]$ in the next three months and finally to $5[1.5,13]$ in the final months $(P<.002)$. The median visit duration was 10 minutes, with an interquartile range of 6-15 minutes.

In the intention-to-treat analysis, patients in the intervention group took close to $50 \%$ more steps than did the control patients. After adjustment for prehospital activity level, the difference was not statistically significant. The intervention also did not significantly affect the length of stay or discharge disposition (Table 3). In the per protocol analysis, the difference in the step count was significant, even after adjustment. The six-clicks score also significantly increased.

To assess for dose response, we compared outcomes among patients who received no intervention, those who received two or fewer days of intervention, and those who received more than two days of intervention (Table 4). The length of stay was significantly longer in patients with more than two days of in- 
TABLE 3. Primary and Secondary Outcomes in the Intention-to-Treat and Per Protocol Analyses

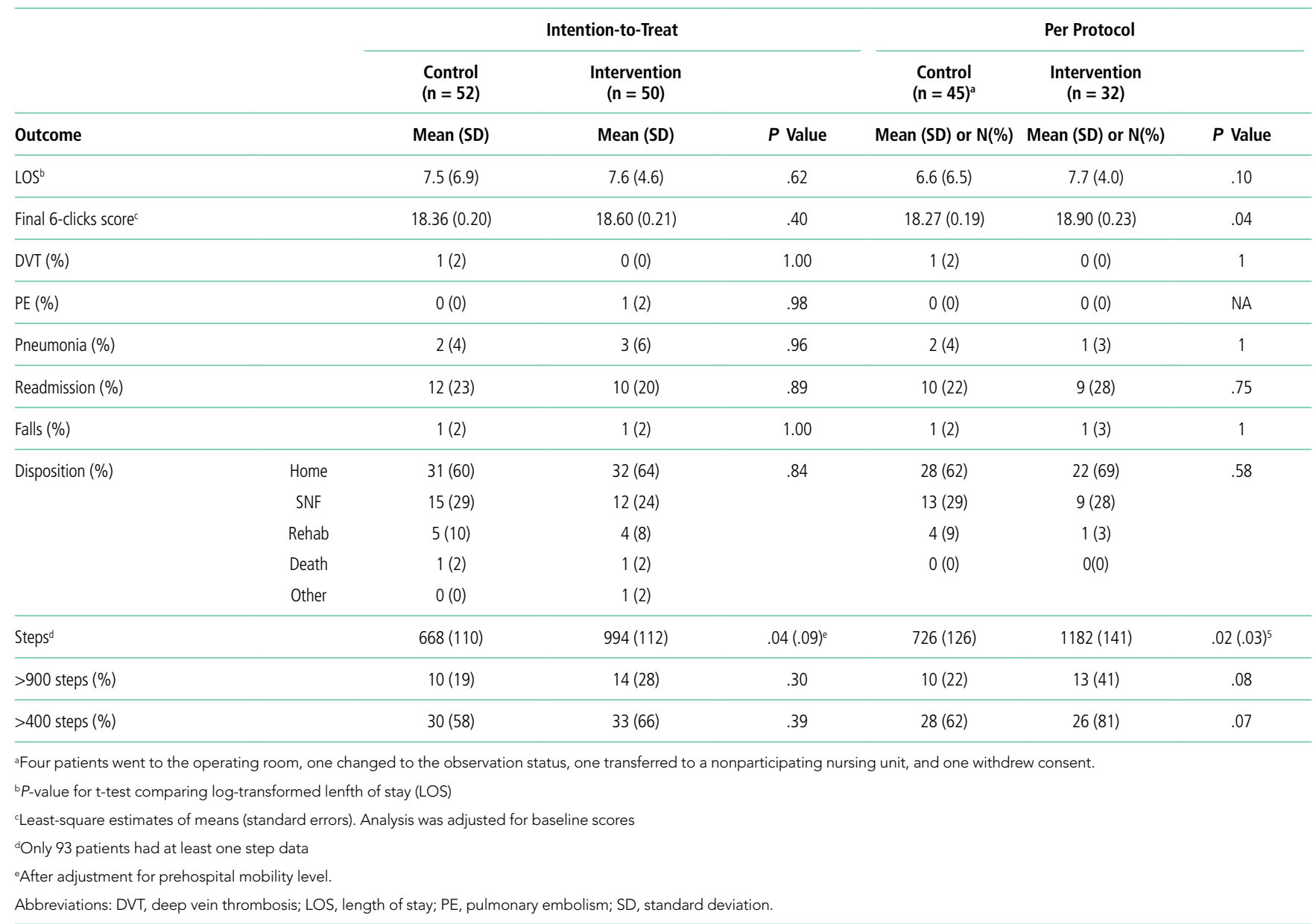

tervention, likely reflecting greater opportunities for exposure to the intervention. The longer intervention time significantly increased the six-clicks score.

We examined the relationship between steps achieved and discharge disposition. Patients who achieved at least 900 steps more often went home than those who did not $(79 \%$ vs. $56 \%, P<$ .05). The ROC for the model of discharge disposition using steps taken as the only predictor had an area under the curve of 0.67 , with optimal discrimination at 411 steps. At a threshold of 400 steps, the model had a sensitivity of $75.9 \%$ and a specificity of $51.4 \%$. Patients achieving 400 steps were more likely to go home than those who did not achieve that goal $(71 \%$ vs. $46 \%, P=.01)$. More patients in the intervention group achieved the 900 step goal $(28 \%$ vs. $19 \%, P=.30)$ and the 400 step goal $(66 \%$ vs. $58 \%$, $P=.39$ ), but neither association reached statistical significance.

\section{DISCUSSION}

In this pilot study conducted with older medical inpatients, we found that assisted ambulation provided by a dedicated mobility technician was feasible and increased the number of steps taken by patients. Not all patients in the treatment group received the intervention partly due to the fact that the program initially did not include weekends and the mobility technicians were sometimes redirected to other nursing duties. Both issues were addressed during the course of the study. In the per protocol analysis, the intervention increased the average six-clicks score and there was a nonsignificant reduction in the percentage of patients discharged to a rehabilitation facility.

A range of hospital-based mobility interventions have been described. Several of which were complex multidisciplinary interventions that included a mobility component. The compliance rates have ranged from $82 \%$ to $93.7 \%, 12,13$ although a systematic review noted that many studies do not provide this level of information. ${ }^{11}$ Interventions were carried out by nursing staff and PT with support from family members and social workers. ${ }^{33-35}$ Ambulation-specific programs have also relied on nurses and PT13,14,36 and, occasionally, on research assistants to implement assisted ambulation protocols. ${ }^{12,37} \mathrm{~A}$ recent study that employed research assistants to deliver inhospital ambulation reported achieving $51.3 \%$ of intended walks. ${ }^{37}$

In contradistinction to previous studies, we created a new role, employing PCNAs as dedicated mobility technicians. We did this for two reasons. First, the approach is less expensive than deploying registered nurses or PTs to ambulate patients and therefore more likely to be adopted by hospitals, especially if it can decrease the cost of an episode of care by avoiding 
TABLE 4. Comparison of Outcomes between Control and Intervention Groups without Days Visited, with up to Two Days of Intervention, and with More than Two Days of Intervention

\begin{tabular}{|c|c|c|c|c|c|}
\hline & & $\begin{array}{l}\text { Control } \\
n=65\end{array}$ & $\begin{array}{c}\leq 2 \text { days } \\
n=22\end{array}$ & $\begin{array}{c}2+\text { days } \\
n=15\end{array}$ & \\
\hline & & Mean (SD) & Mean (SD) & Mean (SD) & $P$ Value for Trend \\
\hline LOS & & $6.94(6.37)$ & $6.63(3.96)$ & $11.4(4.31)$ & .03 \\
\hline Final 6-clicks score ${ }^{\mathrm{a}}$ & & $18.27(0.18)$ & $18.51(0.30)$ & $19.34(0.37)$ & $<.001$ \\
\hline DVT & & $1(2)$ & $0(0)$ & $0(0)$ & .49 \\
\hline PE & & $1(2)$ & $0(0)$ & $0(0)$ & .49 \\
\hline Pneumonia & & $3(5)$ & $2(9)$ & $0(0)$ & .73 \\
\hline Readmission & & $13(20)$ & $8(36)$ & $1(7)$ & .69 \\
\hline Falls & & $1(2)$ & $0(0)$ & $1(7)$ & .34 \\
\hline \multirow[t]{5}{*}{ Disposition } & Home & $39(60)$ & $14(64)$ & $10(67)$ & .60 \\
\hline & SNF & $17(26)$ & $7(32)$ & $3(20)$ & \\
\hline & Rehab & $7(11)$ & $1(5)$ & $1(7)$ & \\
\hline & Death & $1(2)$ & $0(0)$ & $1(7)$ & \\
\hline & Other & $1(2)$ & $0(0)$ & $0(0)$ & \\
\hline >900 steps per day & & $11(17)$ & $6(27)$ & $7(47)$ & 0.01 \\
\hline Steps per day & & $670(100)$ & 904 (164) & $1273(184)$ & 0.004 \\
\hline
\end{tabular}

aLeast-square estimates of means (standard errors). Analysis was adjusted for baseline scores.

Abbreviations: DVT, deep vein thrombosis; LOS, length of stay; PE, pulmonary embolism; SD, standard deviation.

subsequent inpatient rehabilitation. Mobility technicians have no degree or certification requirements and are therefore paid less than nurses or physical therapists. Second, by having a single responsibility, mobility technicians were more likely to engage in their task than nurses, who have competing responsibilities. However, when nurse staffing was short, nurse managers were tempted to recall the PCNAs for other nursing duties. It took time before PCNAs and supervisors prioritized this new responsibility. When they did, the number of attempted walks increased substantially, but the percentage of successful attempts remained constant at 56\%, highlighting the difficulty of getting hospitalized patients to walk.

On average, patients who received the intervention engaged in 72 minutes of additional physical activity and averaged 990 steps per day. Observational data suggest patients accrue about 1,100 steps in the day before discharge, with older patients accruing closer to 900.21 One study found that older patients with fewer than 900 steps per day were likely to experience a functional decline. 32 We also found that patients who achieved at least 900 steps were more likely to go home. However, we found that a lower threshold, namely, 400 steps, offered better discrimination between patients who go home and those who do not. Future prospective studies are needed to establish the appropriate goal for exercise interventions. A lower step goal could dramatically enhance the efficiency of the intervention.

A Cochrane review found that pooled analysis of multidisciplinary interventions that included exercise, often in the form of walking, achieved a small but significant increase in the pro- portion of patients discharged to home (RR $1.08,95 \% \mathrm{Cl} 1.03$ to 1.14). ${ }^{11}$ We found no significant change in the discharge disposition, but our study was underpowered for this endpoint. The six-clicks score showed a small but significant change in the per protocol analysis. The six-clicks score has been shown to correlate with discharge disposition, ${ }^{28,29}$ and an improvement in the score suggests that discharge disposition may be influenced.

The intervention may also not have been implemented for long enough. On average, visits were achieved for one-third of the hospital stay partly because of the delay in PT evaluation, which we required for eligibility. In practice, PT evaluation can occur just a few days before the anticipated discharge. We observed a dose dependent response among patients in the intervention group, suggesting that earlier intervention could be more effective. Earlier intervention might be achieved if the MT performed the six-clicks on potentially eligible patients.

The effects of hospitalization on mobility may be the most pronounced in the long term; one study found that $40 \%$ of hospitalized older patients manifested new ADL or IADL disability three months after discharge compared with $31 \%$ at discharge. 7 Hospital-based mobility interventions may continue to affect subjects' independence for weeks or months. In one RCT, an inpatient ambulation intervention improved mobility in the community one month after discharge. ${ }^{37} \mathrm{~A}$ hospital-based exercise program that included ambulation achieved better functional outcomes one month later. ${ }^{13}$ One RCT that combined inpatient exercise with outpatient care coordination also decreased readmission rates. ${ }^{34}$ We found 
that the intervention did not affect readmission.

This pilot study has several limitations. The sample size was small, and the findings need to be replicated in a larger randomized controlled trial. This is particularly important because the two study arms were not balanced in terms of their prehospital activity. After adjustment for prehospital activity, the differences in the step count in the intention-to-treat analysis were no longer significant. As we adjusted the intervention to hospital workflow, the intervention changed over time. The intention-to-treat analysis may therefore underestimate the effect of the intervention. This work provides a basis for future trial. Finally, discharge disposition depends on a complex interplay of factors, including social factors and preferences, which may not be affected by a mobility intervention.

In summary, an inhospital mobility protocol of attempting ambulation delivered by dedicated mobility technicians three times daily successfully increased the daily step counts and mobility scores of the patients. Studies with a larger sample size are needed to determine whether the proposed approach can affect length of hospital stay, discharge disposition, and overall cost of an episode of care.

Disclosures: Mary Stilphen reports consulting for CreCare and Adeo, which license and distribute AM-PAC short forms, including 6 clicks. All other authors report no conflicts of interest.

Funding: This study was supported by a Research Program Committee grant from the Cleveland Clinic.

\section{References}

1. National Center for Health Statistics. National Hospital Discharge Survey. 2010

2. Corcoran PJ. Use it or lose it--the hazards of bed rest and inactivity. West $J$ Med. 1991;154(5):536-538.

3. Gillick MR, Serrell NA, Gillick LS. Adverse consequences of hospitalization in the elderly. Soc Sci Med. 1982;16(10):1033-1038. doi: 10.1016/0277 9536(82)90175-7.

4. Hirsch $\mathrm{CH}$, Sommers L, Olsen A, Mullen L, Winograd CH. The natural histo ry of functional morbidity in hospitalized older patients. J Am Geriatr Soc. 1990;38(12):1296-1303. doi: 10.1111/j.1532-5415.1990.tb03451.x.

5. Zisberg A, Shadmi E, Sinoff G, Gur-Yaish N, Srulovici E, Admi H. Low mobility during hospitalization and functional decline in older adults. J Am Geriat Soc. 2011;59(2):266-273. doi: 10.1111/j.1532-5415.2010.03276.x.

6. Heit JA, Silverstein MD, Mohr DN, Petterson TM, O'Fallon WM, Melton LJ, 3rd. Risk factors for deep vein thrombosis and pulmonary embolism: A population-based case-control study. Arch Intern Med. 2000;160(6):809-815. doi: 10.1067/mob.2001.107919.

7. Sager MA, Franke T, Inouye SK, et al. Functional outcomes of acute medical illness and hospitalization in older persons. Arch Intern Med. 1996;156(6):645652. doi: 10.1001/archinte.1996.00440060067008.

8. Campbell AJ, Borrie MJ, Spears GF. Risk factors for falls in a community-based prospective study of people 70 years and older. J Gerontol. 1989;44(4):M112-M117. doi: 10.1093/geronj/44.4.M112.

9. Fisher SR, Kuo YF, Graham JE, Ottenbacher KJ, Ostir GV. Early ambulation and length of stay in older adults hospitalized for acute illness. Arch Intern Med. 2010;170(21):1942-1943. doi: 0.1001/archinternmed.2010.422.

10. Graf C. Functional decline in hospitalized older adults. Am J Nurs 2006;106(1):58-67, quiz 67-58

11. de Morton NA, Keating JL, Jeffs K. Exercise for acutely hospitalised old er medical patients. Cochrane Database Syst Rev. 2007(1):CD005955. doi: 10.1002/14651858.CD005955

12. Jones CT, Lowe AJ, MacGregor L, Brand CA, Tweddle N, Russell DM. A randomised controlled trial of an exercise intervention to reduce functional decline and health service utilisation in the hospitalised elderly. Australas $J$ Ageing. 2006;25(3):126-133. doi: 10.1111/j.1741-6612.2006.00167.x.

13. Siebens $H$, Aronow $H$, Edwards D, Ghasemi $Z$. A randomized controlled trial of exercise to improve outcomes of acute hospitalization in older adults. J Am
Geriatr Soc. 2000;48(12):1545-1552. doi: 10.1111/j.1532-5415.2000.tb03862.x.

14. Mundy LM, Leet TL, Darst K, Schnitzler MA, Dunagan WC. Early mobilization of patients hospitalized with community-acquired pneumonia. Chest. 2003;124(3):883-889. doi: 10.1378/chest.124.3.883.

15. Brown CJ, Redden DT, Flood KL, Allman RM. The underrecognized epidemic of low mobility during hospitalization of older adults. J Am Geriatr Soc. 2009;57(9):1660-1665. doi: 10.1111/j.1532-5415.2009.02393.x.

16. Callen BL, Mahoney JE, Grieves CB, Wells TJ, Enloe M. Frequency of hallway ambulation by hospitalized older adults on medical units of an academic hospital. Geriatr Nurs. 2004;25(4):212-217. doi: 10.1016/j.gerinurse.2004.06.016.

17. Fisher SR, Goodwin JS, Protas EJ, et al. Ambulatory activity of older adults hospitalized with acute medical illness. J Am Geriatr Soc. 2011;59(1):91-95. doi: 10.1111/j.1532-5415.2010.03202.x

18. McVey LJ, Becker PM, Saltz CC, Feussner JR, Cohen HJ. Effect of a geriatric consultation team on functional status of elderly hospitalized patients: A randomized, controlled clinical trial. Ann Intern Med. 1989;110(1):79-84. doi:

19. Said CM, Morris ME, Woodward M, Churilov L, Bernhardt J. Enhancing physical activity in older adults receiving hospital based rehabilitation: A phase II feasibility study. BMC Geriatr. 2012;12:26. doi: 10.1186/1471-2318-12-26.

20. Timmerman RA. A mobility protocol for critically ill adults. Dimens Crit Care Nurs. 2007;26(5):175-179; quiz 180-171. doi: 10.1097/01.DCC.0000286 816.40570.da.

21. Sallis R, Roddy-Sturm Y, Chijioke E, et al. Stepping toward discharge: Level of ambulation in hospitalized patients. J Hosp Med. 2015;10(6):384-389. doi: 10.1002/jhm.2343

22. Inouye SK, Wagner DR, Acampora D, Horwitz RI, Cooney LM, Jr., Tinetii ME. A controlled trial of a nursing-centered intervention in hospitalized elderly medical patients: The yale geriatric care program. J Am Geriatr Soc. 1993:41(12):1353-1360. doi: 10.1111/j.1532-5415.1993.tb06487.x.

23. Kalisch BJ, Landstrom GL, Hinshaw AS. Missed nursing care: A concept analysis. J Adv Nurs. 2009;65(7):1509-1517. doi: 10.1111/j.1365-2648.2009.05027.x.

24. Kalisch BJ, Tschannen D, Lee H, Friese CR. Hospital variation in missed nursing care. Am J Med Qual. 2011;26(4):291-299. doi: 10.1177/1062860610395929.

25. Haley SM, Coster WJ, Andres PL, et al. Activity outcome measurement for postacute care. Med Care. 2004:42(1 Suppl):149-161. doi: 10.1097/01. mlr.0000103520.43902.6c.

26. Jette DU, Stilphen M, Ranganathan VK, Passek SD, Frost FS, Jette AM. Validity of the AM-PAC "6-Clicks" inpatient daily activity and basic mobility short forms. Phys Ther. 2014;94(3):379-391. doi: 10.2522/ptj.20130199.

27. Jette DU, Stilphen M, Ranganathan VK, Passek S, Frost FS, Jette AM. Interrater reliability of AM-PAC 6-Clicks" basic mobility and daily activity short forms. Phys Ther. 2015;95(5):758-766. doi: 10.2522/ptj.20140174

28. Jette DU, Stilphen M, Ranganathan VK, Passek SD, Frost FS, Jette AM. AM-PAC "6-Clicks" functional assessment scores predict acute care hospital discharge destination. Phys Ther. 2014;94(9):1252-1261. doi: 10.2522/ptj.20130359.

29. Menendez ME, Schumacher CS, Ring D, Freiberg AA, Rubash HE, Kwon YM. Does "6-Clicks" day 1 postoperative mobility score predict discharge disposition after total hip and knee arthroplasties? J Arthroplasty. 2016;31(9):19161920. doi: 10.1016/j.arth.2016.02.017.

30. Feehan LM, Geldman J, Sayre EC, et al. Accuracy of fitbit devices: Systematic review and narrative syntheses of quantitative data. JMIR Mhealth Uhealth. 2018;6(8):e10527-e10527. doi: 10.2196/10527.

31. Treacy D, Hassett L, Schurr K, Chagpar S, Paul SS, Sherrington C. Validity of different activity monitors to count steps in an inpatient rehabilitation setting. Phys Ther. 2017;97(5):581-588. doi: 10.1093/ptj/pzx010.

32. Agmon M, Zisberg A, Gil E, Rand D, Gur-Yaish N, Azriel M. Association between 900 steps a day and functional decline in older hospitalized patients. JAMA Intern Med. 2017;177(2):272-274. doi: 10.1001/jamainternmed.2016.7266.

33. Counsell SR, Holder CM, Liebenauer LL, et al. Effects of a multicomponent intervention on functional outcomes and process of care in hospitalized older patients: a randomized controlled trial of Acute Care for Elders (ACE) in a community hospital. J Am Geriatr Soc. 2000;48(12):1572-1581. doi: 10.1111/ j.1532-5415.2000.tb03866.x.

34. Courtney M, Edwards H, Chang A, Parker A, Finlayson K, Hamilton K. Fewer emergency readmissions and better quality of life for older adults at risk of hospital readmission: a randomized controlled trial to determine the effectiveness of a 24-week exercise and telephone follow-up program. J Am Geriatr Soc. 2009;57(3):395-402. doi: 10.1111/j.1532-5415.2009.02138.x.

35. Landefeld CS, Palmer RM, Kresevic DM, Fortinsky RH, Kowal J. A randomized trial of care in a hospital medical unit especially designed to improve the functional outcomes of acutely ill older patients. N Engl J Med. 1995:332(20):1338-1344 doi: 10.1056/NEJM199505183322006.

36. Hoyer EH, Friedman M, Lavezza A, et al. Promoting mobility and reducing length of stay in hospitalized general medicine patients: A quality-improvement project. J Hosp Med. 2016:11(5):341-347. doi: 10.1002/jhm.2546.

37. Brown CJ, Foley KT, Lowman JD, Jr., et al. comparison of posthospitalization function and community mobility in hospital mobility program and usual care patients: a randomized clinical trial. JAMA Intern Med. 2016;176(7):921-927. doi: 10.1001/jamainternmed.2016.1870. 